\title{
ÉVOLUTION DES QUANTITÉS D'ALIMENT INGÉRÉES PAR LES BREBIS A LA FIN DE LA GESTATION ET AU DÉBUT DE LA LACTATION. INCIDENCES SUR LEURS PERFORMANCES.
}

\author{
I. - ÉTUDE DE DEUX RATIONS \\ A BASE DE FOIN DE QUALTTÉ DIFFÉRENTE
}

M. TISSIER, M. THÉRIEZ et G. MOLÉNAT

avec la collaboration technique de M. Dumont-St-Priest et A. Torecildas

Station de Rechorches sur l'Elevage des Ruminants, Centre de Recherches de Clermont-Ferrand I. N. R. A., Theix, Saint Genès Champanelle, 63110 Beaumont

\section{RÉSUMÉ}

Au cours de deux expériences successives, des brebis ont été nourries en cases individuelles pendant les 5 dernières semaines de la gestation et les 5 premières semaines de la lactation. Elles ont reçu du foin à volonté, de qualité médiocrc (cxpérience $\mathrm{I}$ : logement sous un hangar ouvert) ou de qualité moyenne (expérience 2 : logement dans un hangar fermé) ainsi qu'une quantité fixe d'aliment concentré (200 g à la fin de la gestation et 4 oo $\mathrm{g}$ au début de la lactation).

La consommation volontaire de foin augmente jusqu'à la $3^{\mathrm{e}}$ semaine avant la mise bas. Elle diminue ensuite jusqu'à l'agnelage, sauf dans l'expérience I où la consommation de foin augmente brusquement au cours de la dernière semaine en liaison avec une diminution importante de la température extéricure. Pendant les 5 premières semaines de la lactation, l'augmentation de consommation de foin est lente ( + I 7 p. Ioo par rapport à la première semaine).

Les principaux facteurs de variation des quantités de foin ingérées sont le poids (liaison consommation de foin-poids vif : $0,3<\gamma<0,4)$, le nombre d'agneaux portés, puis allaités, l'âge des brebis.

La qualité du foin n'affecte pas significativement la consommation volontaire de foin. Les apports énergétiques restent néanmoins plus faibles quand les brebis reçoivent le foin de qualité médiocre. Leur production laitière et la croissance de leurs agneaux sont également diminuées, surtout dans le cas des portées doubles.

L'absence de différences sensibles d'ingestion de matière sèche suivant la qualité du foin, nous a conduit à supposer l'action d'autres facteurs. Les effets probables de la température ambiante et de l'état d'engraissement, sans doute différents entre les deux expériences, sont discutés. 


\section{IN'TRODUC'TION}

Les besoins alimentaires de la Brebis pendant les dernières semaines de gestation sont mal connus. La croissance du ou des foetus, faible pendant les 4 premiers mois de la gestation est maximale au cours du dernier mois, suscitant des besoins élevés de nutriments (WALIACE, I948). Par contre, la consommation volontaire d'aliments a tendance à diminuer dans les semaines qui précèdent la mise bas (cf. revue de ForBES, I970). Il peut en résulter un déficit surtout énergétique. Le mêne, au début de la lactation, les dépenses pour la production de lait, élevées par rapport à la consommation, entraînent une mobilisation des réserves corporelles, qui, si elle est trop intense peut affecter la production laitière et l'état de santé des animaux, ainsi que leurs performances de reproduction ultérieure. Ces problèmes se trouvent accentués par l'intensification de la production ovine, en particulier par l'amélioration de la prolificité et l'accélération du rythme d'agnelage. Dans ce contexte, la recherche d'un rationnement adéquat, avant et après la mise bas, avec différents régimes nécessite la connaissance de l'évolution de la consommation de ces rations et des conséquences sur l'état d'engraissement et les performances d'élevage et de reproduction. C'est pourquoi deux expériences ont été conduites en I97I et I972 pour mesurer individuellement les quantités ingérées de régime à base de foin de qualité différente distribuées avant et après la mise bas, en étudiant leurs conséquences sur le poids des agneaux à la naissance, l'évolution du poids, de la production laitière et du taux de certains paramètres sanguins de leurs mères.

\section{MATÉRIEL E'T MÉTHODES}

Pendant la fin de la gestation (5 semaines), et le clébut de la lactation (5 scmaines), les brebis ont reçu du foin de pré à volonté, de qualité médiocre (expérience I) ou moyenne (expérience 2) et une quantité fixe d'aliment concentré.

\section{Animaux et alimentation}

Dans chacune des deux expériences, yo brebis cle race Limousine âgées de 18 mois à 8 ans, pesant en moyenne $65 \mathrm{~kg}$ ( 50 à $75 \mathrm{~kg}$ ) ont été logées en cases individuelles ( $\mathrm{r} \mathrm{m} \times \mathrm{r}, 50 \mathrm{~m}$ ) sur litière de paille à partir de la $7^{\mathrm{e}}$ semaine avant la mise bas, lans des hangars ouvert (expérence I) ou fermé (expérience 2). L'expérience I s'est déroulée du $1-2$ au 8-4-I97 I et l'expérience 2 du I7-4 au 26-6-I972. La répartition des classes d'âge correspond à la structure moyenne d'un troupeau avec, par conséquent, une faible proportion de brebis jeunes.

Les foins de pré, à base de graminécs, récoltés au premier cycle de végétation étaient hachés et distribués à volonté 2 fois par jour $(8 \mathrm{~h} 30$ et $16 \mathrm{~h} 30)$. Les quantités offertes étaicnt ajustées chaque semaine pour maintenir la proportion de refus à i 5 p. Ioo environ.

Fn outre, dans l'expérience $t$, le foin de qualité mécliocre a été remplacé à partir de la $4^{\mathrm{e}}$ semaine de lactation par un foin de qualité comparable à celui de l'expérience 2.

Les digestibilités des différentes fractions des foins offerts, mesurées sur brebis vides (expérience I) ou sur mâles castrés (expérience 2) sont rapportées au tableau I. 
L'aliment concentré avait la composition suivante:

\begin{tabular}{lclc} 
& p. I00 & & P. Ioo \\
\cline { 2 - 3 } & - & tourteau d'arachide & 29 \\
mais & I5 & tourteau de lin & I9 \\
blé & Io & mélasse & 2 \\
orge & 7,75 & CMV & I2,25 \\
avoine & 5 & &
\end{tabular}

Il était distribué le matin avant le foin à raison de $200 \mathrm{~g}$ par jour pendant la fin de la gestation et $400 \mathrm{~g}$ par jour après l'agnelage. Il était consommé en totalité.

\section{TABLEAU I}

Digestibilité et valeur alimentaive des foins offerts

Digestibility and nutritive value of hays offered

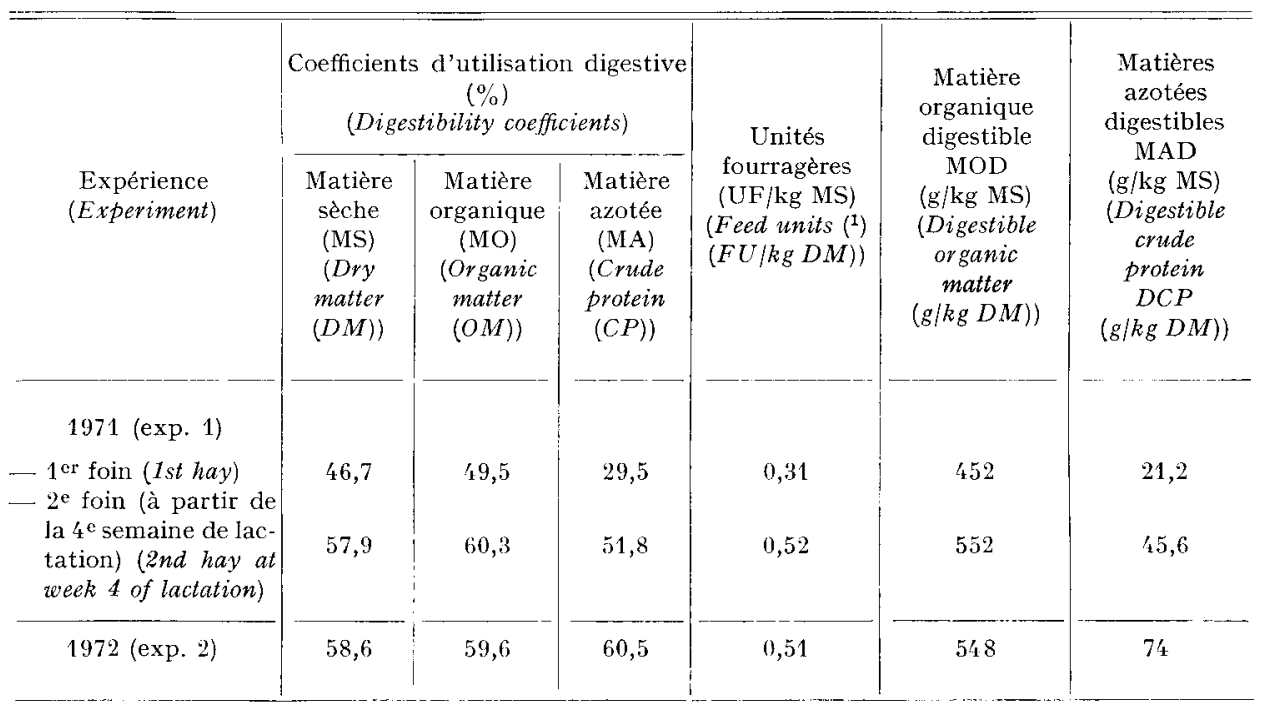

( $\left.{ }^{1}\right) 1$ unité fourragère (UF) $=0,7$ équivalent amidon (EA)

$(1$ feed unit $(F U)=0.7$ starch equivalent $(S E))$.

\section{Mesures}

Les quantités de foin offertes et refusées ont été mesurées chaque jour pendant 5 jours par semaine.

Les teneurs en matière sèche du foin offert et refusé et de l'aliment concentré étaient déterminées deux fois par semaine.

Le poids des brebis a été enregistré au début et à la fin des périodes expérimentales, avant et après la mise bas, et à intervalle de 2 semaines au cours des périodes expérimentales. Les agneaux étaient pesés à la naissance, puis toutes les semaines.

La production laitière de $\mathrm{I} 2$ brebis par expérience $(6$ bessonnières et 6 allaitant un seul agneau) a été estimée un jour chaque semaine par la pesée des agneaux avant et après chacune des 5 tétées espacées de 2 heures (Ricordeau, Boccard et Denamur, ig6o).

Des échantillons de sang ont été prélevés dans la veine jugulaire le matin avant la distribution du repas, au cours des semaines $-4,-\mathrm{I},+2,+4,+6$ par rapport à la mise bas.

Les acides gras non estérifiés (AGNE) du plasma sanguin ont été dosés par la méthode de Dole et Meinertz (1960). 


\section{Interprétation statistique des résultats}

Les consommations hebdomadaires de matière sèche de foin (MSIF), le poids des brebis, le poids des portées, ont été traitées en analyses multidimensionnelles (analyse en composantes principales - analyse factorielle des correspondances). Ces analyses, utilisées dans un but descriptif ont notamment permis de retrouver sans hypothèse préalable, les principaux facteurs de variation de la. MSIF. Ensuite, nous avons testé les effets de ces facteurs sur les consommations et l'évolution du poids des brebis et dcs agneaux par une analyse de variance suivant un plan factoriel.

Les données de production laitière ont été également traitées suivant un plan factoriel $2 \times 2$ (effets de l'année et de l'importance de la portée). Les teneurs moyennes de sang en AGNE ont été comparées suivant l'année et l'importance de la portée.

\section{RÉSULTA TS}

Dans les deux expériences nous avons éliminé 8 animaux pour des raisons diverses (brebis vides, mort ou maladie du ou des agneaux).

De plus, le diagnostic de gestation à l'aide de béliers munis de harnais marqueurs s'est révélé imprécis et beaucoup de brebis ont agnelé plus tôt que prévu. Ainsi, nous ne disposons de données complètes de MSIF et de poids vif que de la semaine - -3 à la semaine +5 , sur 64 femelles seulement. Les calculs statistiques portent sur ces résultats, et les données existantes pour les semaines -4 et -5 sont utilisées dans les figures pour indiquer les tendances.

Les quelques brebis portant 3 ou 4 fœetus n'ont allaité que I ou 2 agneaux, et ont été incluses dans le groupe correspondant.

\section{Quantités de matière sèche ingérées}

\section{- Évolution suivant le stade physiologique.}

Pendant les dernières semaines de la gestation, les quantités de fourrages ingérées augmentent jusqu'à la semaine -3 , puis diminuent au cours de la semaine -2 . Dans l'expérience 2, la diminution continue jusqu'à l'agnelage tandis que dans l'expérience $I$, une augmentation importante des quantités ingérées intervient au cours de la semaine précédant la mise bas, probablement en liaison avec une diminution importante de la température extérieure qui varie alors entre -5 et $-\mathrm{I} 5^{\circ} \mathrm{C}$.

Dès la première semaine de lactation, malgré l'augmentation de $200 \mathrm{~g} / \mathrm{j}$ de $1 \mathrm{a}$ quantité d'aliment concentré distribué, les brebis consomment Io à I 5 p. Ioo de plus de fourrages que lors de la dernière semaine de gestation (en se référant aux consommations des brebis de 1'expérience 2 pendant cette dernière semaine).

L'ingestion n'augmente que lentement au cours des 4 premières semaines de lactation puis s'accroît à la $5^{\mathrm{e}}$ semaine. L'augmentation totale des quantités ingérées est en moyenne pour l'ensemble des brebis de $\mathrm{I} 6,7 \mathrm{p}$. Ioo par rapport à la première semaine.

L'étude de la consommation de matière sèche de foin, au cours des jours qui précèdent et stivant l'agnelage, montre une grande variabilité d'un jour à l'autre et la diminution importante des quantités ingérées à la veille de la mise bas. La consommation augmente de nouveau très rapidement après l'agnelage. 
Facteurs de variation des quantités de foin ingérées

- Qualité du foin (fig. I).

Malgré la différence marquée entre les digestibilités et les teneurs en matières azotées des foins des 2 essais, les consommations sont identiques pendant la fin de la gestation, sauf au cours de la dernière semaine.

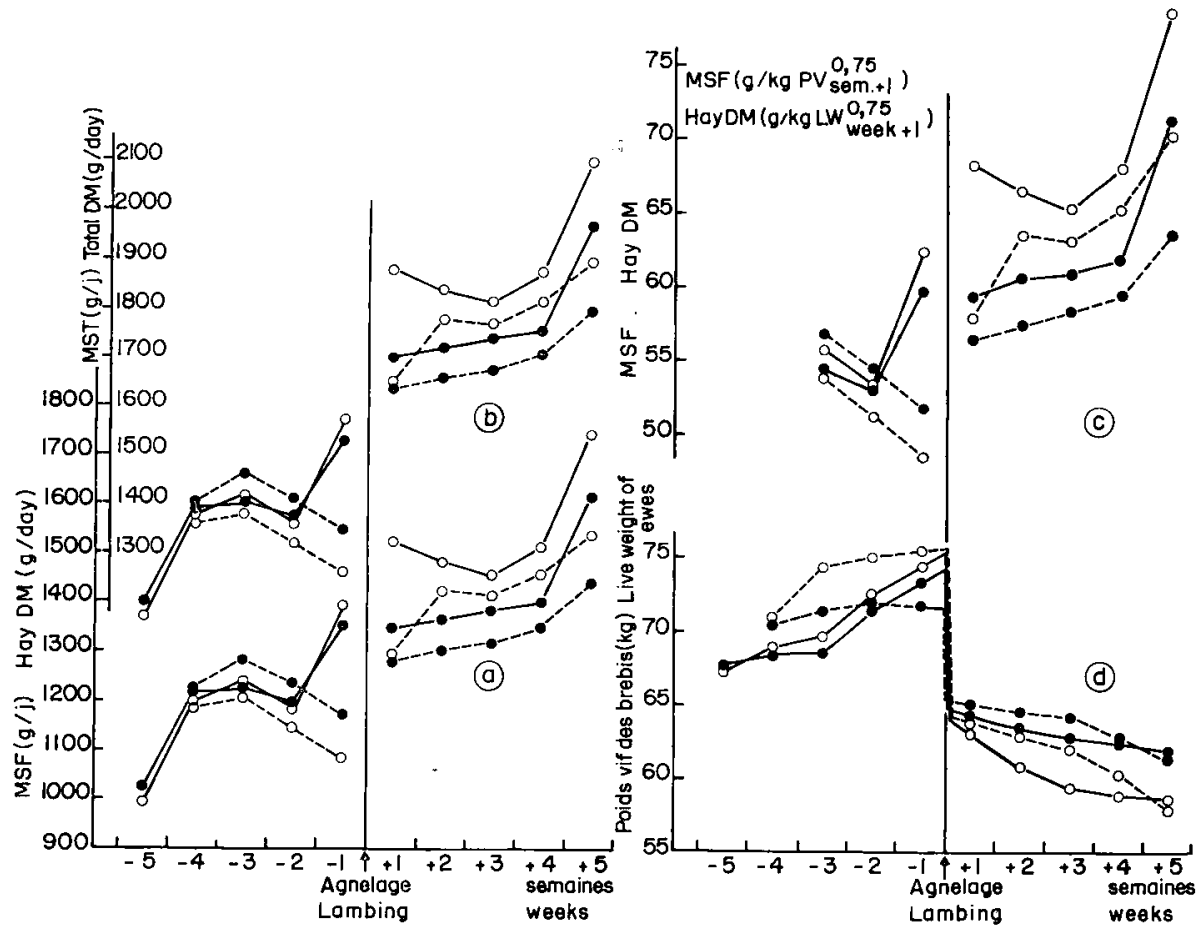

Fig. I. - Influence de la qualité du foin et de l'importance de la portée sur:

a) la consommation de matière sc̀che de foin par brebis et par jour

c) l'évolution du poids vif des brebis.

Effect of the quality of hay and litter size on:

a) hay dry matter intake per ewe per day

b) total dry matter intake per ewe per day

c) hay dry matter intake per $\mathrm{kg}$ metabolic body weight

d) bodly weight changes of the ewes
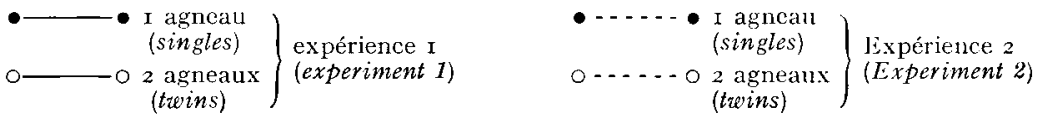

Au début de la lactation (3 premières semaines), les brebis recevant le foin de qualité médiocre ingèrent autant, voire plus, de fourrage que celles qui reçoivent le foin de qualité moyenne. Par ailleurs, leur augmentation de consommation à partir de la $4^{\mathrm{e}}$ semaine de lactation est également plus élevée, bien que la qualité des foins soit alors comparable (différence significative $\mathrm{P}<0$,OI). 
Il faut noter ici que pour les brebis de l'expérience I, logées dans un hangar ouvert, la température suit approximativement celle de l'extérieur, elle-même en moyenne plus basse qu'au cours de l'expérience 2, comme le montre la figure 2. Nous n'avons pas relevé la température à l'intérieur du hangar fermé ; nos observations nous permettent cependant de penser que la température ambiante était de 3 à $4^{\circ} \mathrm{C}$ supérienre à celle de l'extérieur lorsque celle-ci varie entre o et $\mathrm{I} 2^{\circ} \mathrm{C}$.

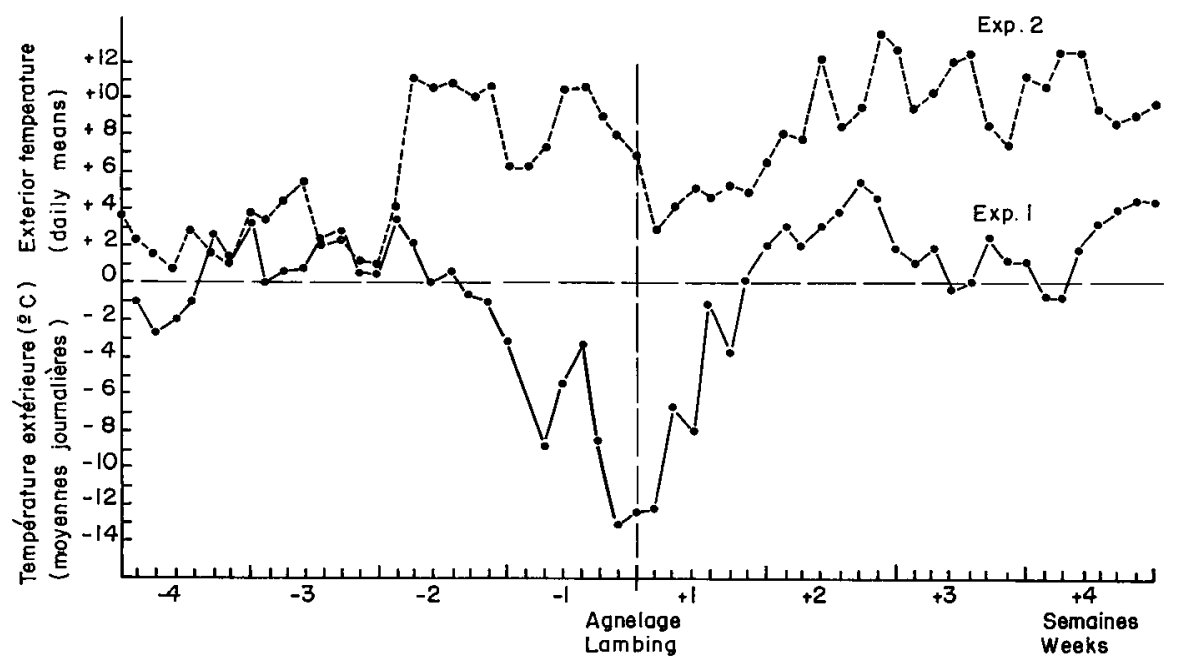

FIG. 2. - Évolution de la température extérieure au cours des 2 expériences (moyennes journalières)

Exterior temperature changes during the 2 experiments (daily means)

Lxpérience I (Experiment 1)

- - Expérience 2 (Experiment 2)

En conséquence, et d'après la majorité des auteurs, si l'effet de la température ambiante existe, non seulement au moment de la mise bas mais durant toute l'expérience $I$, il affecterait les quantités ingérées en sens inverse de la qualité du foin, ce qui peut expliquer l'absence d'effet marqué de cette dernière.

\section{- Poids des brebis.}

La variabilité du poids vif (PV) entre les brebis explique une partie de celle de la MSIF comme le montre le coefficient de corrélation de 0,3 à $0,4(\mathrm{P}<0, \mathrm{OI})$ entre ces deux variables. La liaison la plus élevée est observée pendant la lactation.

Ainsi, les brebis les plus lourdes consomment plus de foin. On peut éliminer en grande partie cet effet du poids en exprimant les quantités ingérées par $\mathrm{kg}$ de poids métabolique $\left(\mathrm{PV}^{0,75}\right)$ (ce critère est bien représenté sur 1'un des axes principaux des analyses en composantes principales ou factorielles des correspondances). Nous avons choisi le poids métabolique à la semaine $+\mathrm{I}$, qui semble le moins perturbé par la croissance des foetus, ou le poids des contenus digestifs. 
- Nombre d'agneaux portés puis allaités (fig. I).

Les brebis portant 2 foetus ( 28 animaux) ont tendance à consommer moins de foin en fin de gestation que celles qui n'en portent qu'un (36 animaux) ( $200 \mathrm{~g} / \mathrm{j}$ contre I 242 en moyenne pendant les 3 dernières semaines de gestation, différence NS). Au contraire, les bessonnières ont la consommation la plus élevée au début de la lactation ( $480 \mathrm{~g} / \mathrm{j}$ contre I 375 en moyenne pendant les 5 premières semaines de lactation, différence NS). Cette différence devient significative lorsque la consommation est rapportée au poids métabolique à la semaine $+\mathrm{I}(\mathrm{P}<0,05)$.

- Age des brebis (fig. 3).

Le nombre de brebis jeunes étant restreint, l'influence du facteur âge est difficile à préciser. Néanmoins, nous avons pu dégager des tendances intéressantes.
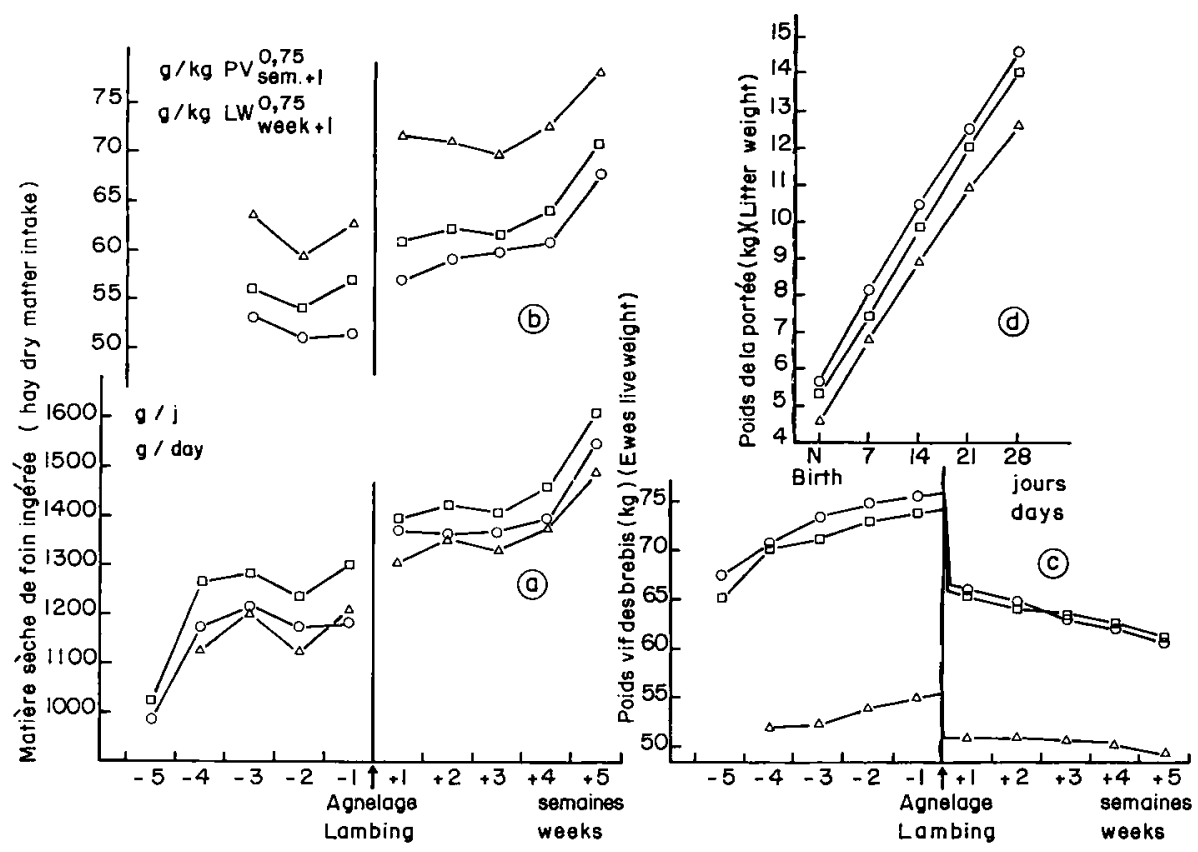

FIG. 3. - Effet de l'âge des brebis sur:

a) leur consommation de matière sèche de foin/brebis/jour
b)
- /kg de poids métabolique/jour
c) l'évolution de leur poids vif
d) l'évolution du poids de leur portée

Effect of erves age on:

a) hay dry matter intake/ewe/day

b) - - - - $/ \mathrm{kg}$ metabolic body weight/day

c) ewes body weight changes

d) litter body weight changes

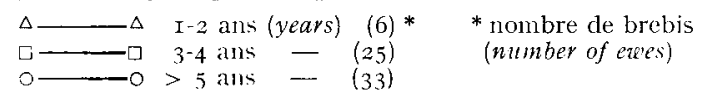


Les brebis adultes (âge $>3$ ans) ingèrent en moyenne 7 à $8 \mathrm{p}$. Ioo de plus que les jeunes en liaison avec leur poids plus élevé mais, la différence n’est pas significative. Par contre, les quantités ingérées rapportées au poids métabolique diminuent quand 1'âge augmente, aussi bien pendant la fin de la gestation qu'au début de la lactation (en moyenne 72,$6 ; 63,8$ et $60,8 \mathrm{~g} / \mathrm{kg} \mathrm{PV}^{0,75}$ respectivement pour les classes d'âge I-2 ans, 3-4 ans, 5 ans et plus, pendant les 5 premières semaines de lactation, $\mathrm{P}<0,05)$.

\section{- Variations individuelles non imputables aux facteurs précédents.}

Le poids des brebis et la taille de leur portée ne rendent compte approximativement que de 27 à $45 \mathrm{p}$. Ioo de la variabilité de la MSIF suivant le stade physiologique (les plus fortes valeurs sont observées pendant la lactation). Par ailleurs, 1'âge de ces animaux et le facteur année n'ont qu'une importance limitée. On peut donc estimer, grâce à l'analyse des données, que 40 à 50 p. Ioo de la variabilité de la MSIF est due à des variations individuelles non imputables aux facteurs identifiés précédemment.

\section{Conséquences sur les performances et l'état de santé des animaux}

\section{- Évolution du poids des brebis (fig. I, tab1. 2).}

Le tableau 2 indique le poids des brebis au voisinage de l'agnelage et les variations de poids au cours de la gestation et de la lactation suivant la qualité du foin et la taille de la portée. Le gain de poids moyen de l'ensemble des brebis au cours des 5 dernières semaines de gestation (PV $-\mathrm{I}$ moins $\mathrm{PV}-5)$ est de $5,8 \mathrm{~kg}( \pm 3,3)$, soit $8,8 \mathrm{p}$. Ioo $( \pm 5, \mathrm{I}) \mathrm{du}$ poids à la semaine - 5. Ce même gain est de $7,8 \mathrm{~kg}( \pm 2,5)$ et $4,4 \mathrm{~kg}( \pm 3)(\mathrm{P}<0,00 \mathrm{I})$ respectivement pour les brebis nourries avec le foin de qualité médiocre et celles nourries avec le foin de qualité moyenne. Ces dernières étant en moyenne plus lourdes que les autres au début de l'expérience et jusqu'à la semaine $-3(\mathrm{P}<0,05)$, les poids des deux groupes à l'approche de la mise bas sont comparables. Pendant la lactation, les pertes de poids des deux groupes sont respectivement de $-3,6 \mathrm{~kg}\left( \pm_{2}, 2\right)$ et $-4,5 \mathrm{~kg}( \pm 2,6)$ pour le foin médiocre et le foin moyen pendant les 5 premières semaines de lactation (non significatif).

Dans l'interprétation de ces variations de poids, il est nécessaire de tenir compte des variations de poids des contenus digestifs, notamment en relation avec les augmentations de consommation importantes observées lors de la dernière semaine de gestation, et de la $5^{\mathrm{e}}$ semaine de lactation dans l'expérience $\mathrm{I}$. D'après des estimations obtenues sur vaches laitières par différents auteurs (JOURNET, communication personnelle), une variation de consommation de I $\mathrm{kg}$ de matière sèche s'accompagne d'une variation de 4 à $5 \mathrm{~kg}$ de poids de contenus digestifs.

On peut donc estimer que compte tenu des augmentations de consommation citées, les variations de poids des brebis de l'expérience I, pendant la fin de la gestation et le début de la lactation, doivent être diminuées de I $\mathrm{kg}$. Ainsi l'évolution du poids reste différente pendant la fin de la gestation entre les deux expériences. Par contre, pendant la lactation, l'évolution du poids serait comparable d'une expérience à l'autre. 


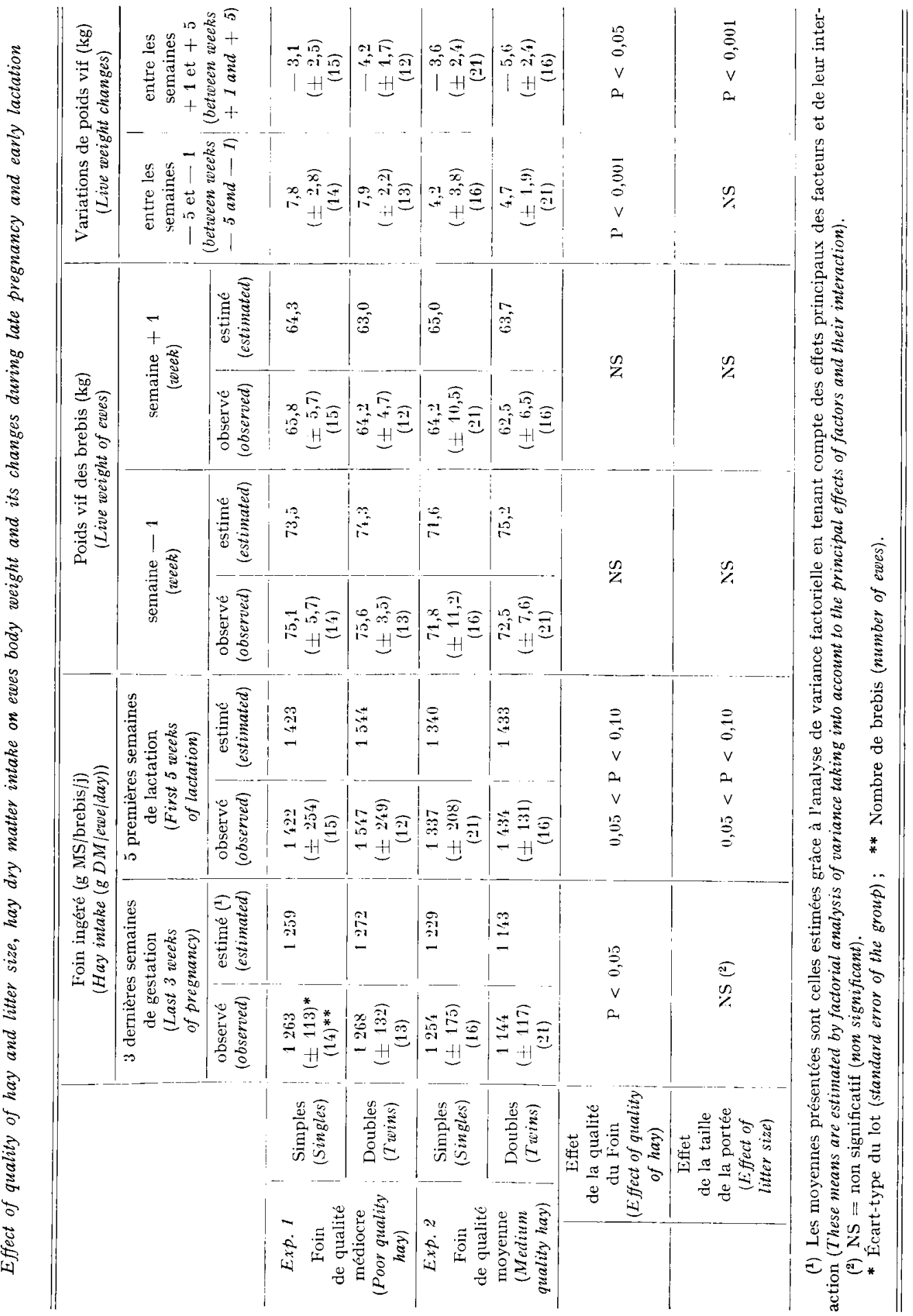


- Evolution de la production laitièro (fig. 4).

La quantité totale de lait produit au cours des 5 premières semaines de la lactation par les bessonnières est de $40 \mathrm{p}$. Ioo environ plus élevée que pour les brebis allaitant un seul agneau. La forme des courbes cle production laitière est également différente. Flles présentent un maximum à la $2^{\circ}$ semaine de lactation et une décroissance rapide pour les premières. Pour les secondes la forme est identique au cours des 3 premières semaines (bien que le niveau maximum soit plus faible), mais la production augmente ensuite au cours des $4^{\mathrm{c}}$ et $5^{\circ}$ semaines.

Les brebis recevant le foin de qualité médiocre ont une production totale de lait au cours des 3 premières semaines de lactation inférieure de $\mathrm{I} 9 \mathrm{p}$. Ioo et I I p. Ioo à celles des brebis de l'expérience 2 , respectivement pour les bessonnières et les brebis allaitant un seul agneau.

\section{- Evolution du poids des agneaux (fig. 4).}

Le poids moyen à la naissance ainsi que la croissance moyenne des portées de o à 28 jours sont légèrement plus élevés lorsque les mères reçoivent le foin de qualité moyenne plutôt que le foin de qualité médiocre : $5,590 \mathrm{~kg}$ contre $5.320(\mathrm{P}<0, \mathrm{I})$ et $323 \mathrm{~g} / \mathrm{j}$ contre $299(\mathrm{P}<\mathrm{O}, \mathrm{OI})$. Ainsi les portées sont à $28 \mathrm{j}$ plus lourdes de $\mathrm{I} \mathrm{kg}$ $(\mathrm{P}<\mathrm{o}, \mathrm{OI})$. La figure 4 montre que ces différences proviennent surtout des agneaux doubles, dont la proportion est la même dans les deux expériences. Leur poids à $28 \mathrm{j}$ et leur croissance de o à $28 \mathrm{j}$ sont affectés lorsque la mère reçoit du foin de qualité médiocre $(8,5 \mathrm{~kg}$ contre 9,3 et $\mathrm{I} 80 \mathrm{~g} / \mathrm{j}$ contre $20 \mathrm{I})$. Par contre, les différences sont très faibles pour les agneaux simples, quelle que soit la qualité du régime de leur mère.

Nous n'avons pas mis en évidence d'effet du sexe des agneaux, bien que la proportion de femelles soit légèrement plus élevée dans l'expérience 2 .

\section{- Teneur du plasma sanguin en acides gras non estérifiés (fig. 4).}

La teneur plasmatique en AGNE augmente au cours du dernier mois de gestation. Elle atteint un niveau élevé dans les deux premières semaines de lactation, puis diminue jusqu'à un niveatı comparable à celui atteint un mois avant mise bas. Ėlle est plus élevée pour les brebis portant 2 fotus, puis allaitant 2 agneaux.

Cette teneur n'est pas modifiée significativement par la qualité du foin à la fin de la gestation. Par contre les brebis recevant le foin de qualité médiocre ont, au début de la lactation, des teneurs en AGNE plus élevées que les autres bien que leur production laitière soit plus faible et leur consommation de MSF plus élevée.

\section{Bilan énergétique au cours des 3 dernières semaines de gestation et des 5 premières semaines de lactation}

Les digestibilités des régimes foin + aliments concentrés n'ont pas été mesurées. Nous avons donc estimé la digestibilité de l'aliment concentré grâce aux tables de KELLNER et considéré les digestibilités des foins et de l'aliment concentré comme associatives étant donné la faible proportion de ce dernier (DEMARQUH,LY, communication personnelle).

Les bases de calcul des besoins des animaux et les réstultats du bilan réalisé en 
énergie métabolisable sont consignés dans le tableau 3 suivant la qualité du foin et la taille de la portée.

Pendant la fin de la gestation (semaines - -3 et - 2), les apports d'énergie dépassent peu les besoins d'entretien ( $+\mathrm{I}_{5}$ à $25 \mathrm{p}$. I 00 ) quand les brebis reçoivent le foin de qualité médiocre. Par contre lorsque le foin est de meilleure qualité, ces apports sont de 30 à $43 \mathrm{p}$. Ioo supérieurs aux besoins d'entretien.
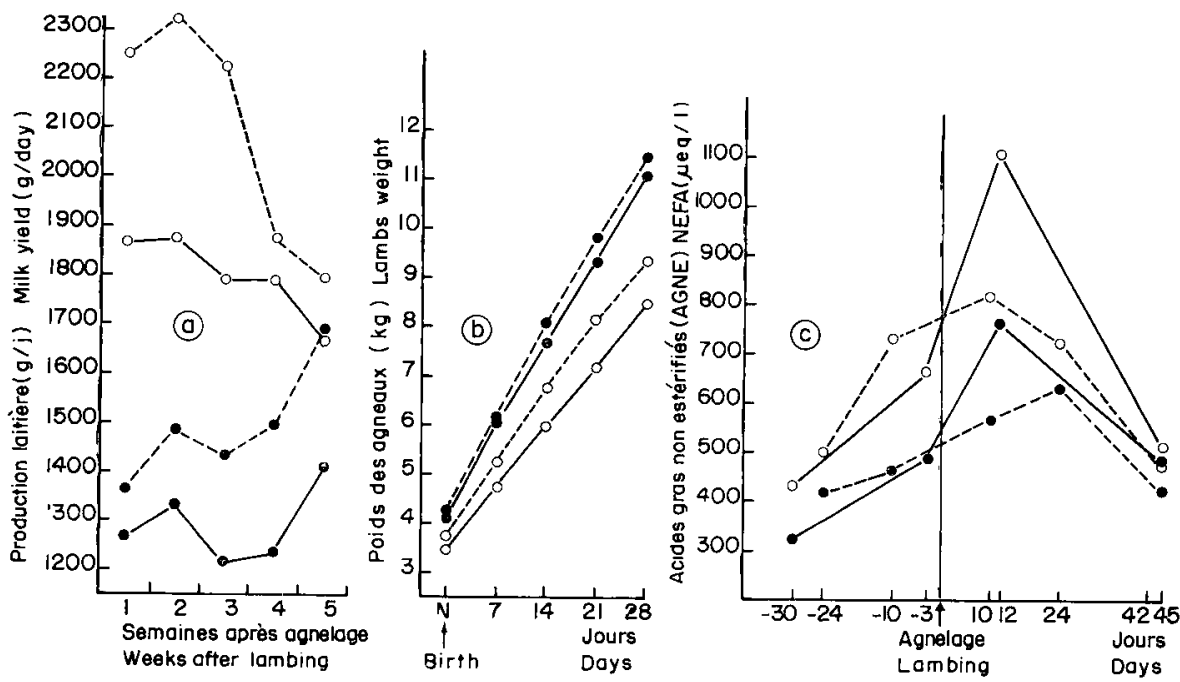

I.IG. 4. - Influence de la qualité du foin et de limportance de la portée sur:

a) la production laiticre

b) le poids des agneaux

c) le taux plasmatique d'acides wras non estérifiés

Effect of hay quality and litter size on:

a) milk yield

b) lambs weight

c) plasma levels of non esterified fatty acids
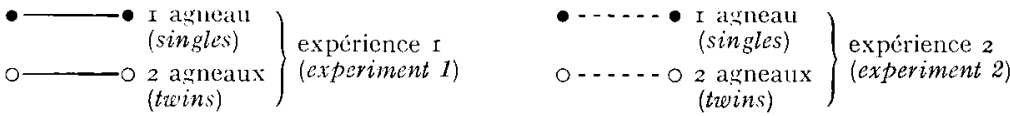

Au début de la lactation où nous avons pu calculer des besoins de production (tabl. 3), le déficit énergétique enregistré entre les apports et les besoins cumulés (entretien + production laitière) est généralement maximum au cours de la $2^{e}$ semaine; il diminue ensuite en liaison avec la baisse de production laitière (surtout pour les bessonnières) et l'augmentation de la consommation de foin.

Pendant les 3 premières semaines de la lactation, les brebis allaitant un seul agneau ont un déficit comparable quelle que soit la qualité du foin, que ce soit en valeur absolue (environ I ooo $\mathrm{kcal} / \mathrm{j}$ ) ou en pourcentage des besoins totaux (entre ro et $25 \mathrm{p}$. I00). Les bessonnières ont un déficit énergétique plus élevé, d'une part en raison de leur production laitière plus forte, d'autre part lorsqu'elles reçoivent le foin de meilleure qualité. Cependant, quand le déficit est exprimé en pourcentage des besoins totaux, il est comparable suivant la qualité du foin : 33 p. Ioo (foin médiocte) et $36 \mathrm{p}$. Ioo (foin moyen) en moyenne pendant les 3 premières semaines de la lactation. 

$+4$ इ

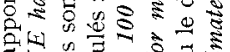

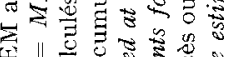
- कु के है ه

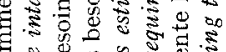
०.

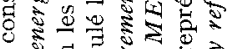
ब

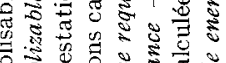
잉

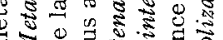
द्व

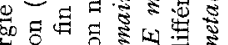

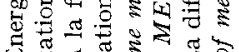

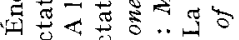

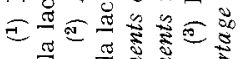
造 


\section{DISCUSSION}

Les résultats de ces deux expériences permettent de dégager des conclusions déjà observées par d'autres auteurs sur les facteurs de variation et l'évolution de la consommation volontaire pendant la fin de la gestation et le début de la lactation.

Parmi les facteurs de variation inter individuelle des quantités de matière sèche de fourrages ingérées, l'importance du poids est limitée. La liaison MSIF-PV trouvée $(0,3<\mathrm{P}<0,4)$ est du même ordre que celle rapportée par JounNET et al. (I965) sur vaches laitières.

L'influence du nombre de fœtus portés et d'agneaux allaités sur les quantités ingérées n'est pas significative, mais les tendances que nous observons sont en accord avec la majorité des travaux. A la fin de la gestation la diminution de consommation des mères portant 2 agneaux est plus importante que celle des mères d'agneaux simples, comme dans les résultats de Forbes (I970), ThÉriez et MolÉnat (I970). Pendant la lactation, les bessonnières ingèrent plus que les brebis allaitant un agneau comme le rapportent HADJIPIERIS et HOLMES (I966). Cette ingestion plus forte est à mettre en relation avec la production laitière plus élevée des bessonnières (WALIACE, I948; RicondeAu et al., I960; PEART, I967, I970). Beaucoup d'auteurs (ForbEs, I970 : a review; JounNeT el al., I965) rapportent que la quantité de MSI ingérée par des vaches laitières augmente avec la quantité de lait produite.

Pour l'effet de l'âge des animaux, il est difficile de conclure vu le faible nombre de brebis jeunes dans les deux essais. Cependant, les brebis d'âge $\leqslant 2$ ans ingèrent plus de matière sèche par $\mathrm{kg}$ de poids métabolique que les brebis plus âgées, et il est probable que ces animaux aient encore des besoins non négligeables pour leur croissance (elles pèsent en moyenne Io $\mathrm{kg}$ de moins que les brebis adultes).

La diminution de la consommation volontaire des fourrages, observée à partir de la $3^{\text {e }}$ semaine avant l'agnelage, a été relatée par différents auteurs sur brebis (HAdjipieris et Holmes, I966 sur vaches; MAkELA, I956; Johnson et al., I966) et sur chèvres (FEHR et al., I97I). Ils l'ont expliqué par des limitations de l'appétit, d'ordre physique (compression du rumen par l'utérus gravide), ou métabolique (augmentation du taux de certaines hormones au fur et à mesure que la gestation avance) (FORBES, I970). Cette diminution reste cependant assez faible puisqu'elle est de l'ordre de Io p. Ioo au cours des 3 dernières samaines de gestation pour l'expérience 2 . On peut calculer une diminution analogue à partir des résultats antérieurs de ThÉRIEZ et Molénat (1970) sur des brebis Limousines également. La préparation de la parturition cause une perturbation importante, mais brève de la consommation puisque celle-ci diminue de ro à $20 \mathrm{p}$. Ioo seulement la veille de la mise bas.

Au début de la lactation, l'augmentation lente des quantités ingérées relativement à celle de la production laitière entraîne un déficit important, malgré la distribution accrue de l'aliment concentré ( $400 \mathrm{~g}$ au lieu de $200 \mathrm{~g}$ pendant la fin de la gestation). La majorité des auteurs cités pat Forbes (I970) et JourNeT et JARRIGE (I970) signalent également le faible appétit des vaches laitières pour les fourrages au début de la lactation et le décalage résultant entre le maximum de besoins $\left(2^{\mathrm{e}}\right.$ semaine après le vêlage) et le maximum de consommation de fourrages (entre le $2^{\text {e }}$ et le $5^{\mathrm{e}}$ mois de lactation). 
En plus de ces résultats, l'analyse de l'influence de la qualité du foin et de l'année, et du bilan énergétique estimé de l'énergie métabolisable nous permet de discuter quelques points.

Généralement une liaison positive est observée entre les quantités de matière sèche de fourrages ingérées et la digestibilité de la ration (BLAXTER et al., I96I ; DEMARQUII, $\mathrm{Y}$ et WEIss, I970 : résultats obtenus sur des moutons). Les résultats de deux expériences montrent que la consommation des brebis recevant le foin de qualité médiocre est comparable, voire supérieure (fig. I) à celle des brebis nourries avec le foin de qualité moyenne. D'autres facteurs que la qualité du foin ont pu jouer, particulièrement la température ambiante et l'état d'engraissement des animaux. Il est probable que la température ait influencé la consommation de matière sèche des brebis de l'expérience 1 , non seulement pendant les périodes voisines de l'agnelage, mais pendant toute la durée de l'expérience. En effet, au moins pendant la lactation,

- on peut penser que la différence de température ambiante entre les deux expériences était de 1'ordre de 7 à $\mathrm{I}^{\circ} \mathrm{C}$. Différents auteurs, notamment WrNFIELd et al. (Ig69) sur brebis et MCDONALD et BEL, (r958) sur vaches laitières ont observé l'augmentation de la consommation volontaire suite à la diminution de la température. Par ailleurs, la tonte des brebis, qui se traduit également par un choc thermique, a la même conséquence (WoDzickA, r963).

En plus de cet effet possible des conditions de milieu, il semble que les brebis nourries avec le foin de qualité moyenne étaient en meilleur état d'engraissement que celles qui ont reçu le foin de qualité médiocre. Plusieurs observations permettent de soutenir cette hypothèse. Les brebis de l'expérience 2 , bien qu'issues du même troupeau, avaient un poids plus élevé de $4 \mathrm{~kg}$ que celles de la première expérience. Cette observation coïncide avec le fait que ces brebis ont bénéficié dans de bonnes conditions d'un pâturage de montagne durant l'été précédent l'expérience 2. Corrélativement, elles ont gagné moins de poids durant les dernières semaines de gestation, malgré leur ration de meilleure qualité. En outre, lorsqu'à partir de la $4^{\mathrm{c}}$ semaine de lactation les brebis de l'expérience I reçoivent un foin de qualité comparable à celui de l'expérience 2 , elles continuent à avoir tune consommation de matière sèche supérieure et maintiennent leur poids. BINES (I97I) sur vaches, FooT-JANET (I970, I972) sur brebis ont trouvé que des animaux gras ingèrent moins que des animaux maigres.

Nos résultats ne permettent pas de mieux préciser l'action de ces facteurs qui sont vraisemblablement à l'origine de l'absence de différences dans les quantités ingérées en fonction de la qualité du foin.

En ce qui concerne le bilan énergétique, d'une façon générale, les apports alimentaires à la fin de la gestation sont faibles par rapport aux besoins d'entretien (entre I 5 et $43 \mathrm{p}$. Ioo) compte tenu de la croissance importante des foctus et de la relative inefficacité de la transformation de l'énergie métabolisable en énergie nette pendant cette période (WALLACE, I948 ; RATTRAY et al, I973-I974). Il est probable que les brebis mobilisent alors leurs réserves corporelles comme le montrerait l'augmentation du taux d'acides gras non estérifiés du plasma. Cette mobilisation est sans doute maximum au cours des 2 à 3 premières semaines de lactation, où le déficit atteint des proportions de l'ordre de $20 \mathrm{p}$. roo et de $35 \mathrm{p}$. roo des besoins calculés respectivement pour les brebis allaitant un seul agneau et les bessonnières.

Les brebis recevant le foin de qualité médiocre sont relativement sous alimentées par rapport aux autres pendant la fin de la gestation. Cette sous-alimentation n'est 
cependant pas très sévère comme en témoignent les taux d'acides gras non estérifiés, qui restent inférieurs à $750 \mu \mathrm{eq} / 1$ même pour les brebis ditoques. D'après RussEI. et al. (I967) ces valeurs indiqueraient l'absence de sous-alimentation sévère et de mobilisation importante des réserves corporelles.

$\mathrm{Au}$ début de la lactation, la valeur absolue du déficit énergétique est, pour les bessonnières, supérieure lorsqu'elles recevaient le foin de qualité moyenne plutôt que le foin de qualité médiocre. Ce résultat est en relation avec la production laitière plus élevée, mais on peut remarquer qu'en pourcentage des besoins totaux, le déficit est comparable quelle que soit la qualité du foin.

Il est possible que la production laitière n'augmente plus à partir d'un certain niveau de déficit par rapport aux besoins, qui constituerait une aptitude à mobiliser les réserves corporelles.

En conclusion, la qualité du foin n'a pas affecté sensiblement les consommations de fourrages, les variations de poids vif des brebis et le poids des agneaux à la naissance, mais des facteurs extérieurs tels que la température ambiante et l'état d'engraissement ont pu jouer un rôle. Néanmoins, la distribution d'un foin de médiocre qualité s'est traduite par une diminution de la vitesse de croissance des agneaux, consécutive à une production laitière plus faible. Cet effet s'est manifesté surtout pour les portées doubles.

Reru pour publication en juin 1975.

\title{
SUMMARY
}

\author{
VARIATION IN THE VOLUNTARY FEED INTAKE \\ OF EWES DURING LATE PREGNANCY AND EARLY LACTATION : \\ INCIDENCES ON THEIR PERFORMANCES. \\ I. - STUDY OF TWO HAY DIETS OF DIFFERENT QUAITTY
}

The factors of variation in the voluntary feed intake of ewes during late pregnancy (5 weeks) and early lactation ( 5 weeks) and their consequences on health and growth of the lambs were studied during two experiments using diets containing meadow hay. The ewes were fed ad libitum with hay of poor quality (experiment I : organic matter cligestibility 49.5. p. 1oo) or moderate quality (experiment 2 : organic matter digestibility $59.6 \mathrm{p}$. Ioo) and with a constant amount of concentrate feed (200 g during late pregnancy, $400 \mathrm{~g}$ during early lactation). The animals were housed in individual pens under open (exp. I) or closed (exp. 2) shed.

Feed intake, weight gain and growth were measured in all the lambs. In addition, the milk yield was recorded in some of the ewes as well as the plasma level of non esterified fatty acids $(A G N E=$ NEFA).

I. The voluntary intake of hay increased up to the 3 rd week prior to lambing. Thereafter it decreased until lambing, except in experiment I where the hay intake increased abruptly during the last week in relation with a large fall in the outer temperature (between -5 and $-\mathrm{I} 5^{\circ} \mathrm{C}$ ). During the first 5 weeks of lactation, the increase in the hay intake was slow $(+$ I 7 p. Ioo as compared to the first week).

2. The main factors of variation in the voluntary intake of hay were the following:

- liveweight of the animals : the heaviest ewes showed a tendency to cat more hay. The correlation coefficient between liveweight and hay matter intake (MSIF $=$ HDMI) ranged between 0.3 and $0.4(\mathrm{P}<0.01)$;

- number of lambs borne, and suckled : the ewes bearing two lambs showed a tendency to eat less hay during the last weeks of pregnancy than those bearing one lamb, especially in experiment 2. On the other hand, during lactation the ewes nursing two lambs ate more hay than those nursing one only lamb; 
- age of the ewes : although the number of young ewes was small in the two experiments, it can be noted that they consumed more hay than the adult ewes, when the amounts ingested were expressed relatively to the metabolic weight ( $\mathrm{g}$ HDMT/ $\mathrm{kg} \mathrm{B} \mathrm{BW}^{\mathbf{0} .75}$ ).

3. Paradoxically, the hay quality, although very different in the two experiments, did not affect very much the hay intake. Parallely, it can be noted that the ambiant temperature conditions were different from one experiment to the other, since the housing conditions of the animals were different (open or closed shed). Thus, the large temperature fall during the last week of pregnancy in experiment I excepted, the ambiant temperature during the whole experiment remained lower than that of experiment 2 .

4. On account of the liveweight gain of the ewes and the possible variations in the weights of digesta (correlated to the variations in the amounts of feed ingested), the weight gain of the cwes represented $6.8 \mathrm{~kg}$ (experiment I) and $4.4 \mathrm{~kg}$ (experiment 2) during the last 5 weeks of pregnancy. During the first 5 weeks of lactation, the weight losses were comparable for the two experiments and ranged about $4.5 \mathrm{~kg}$.

Although the quality of the hay did not affect the hay intake of the animals, their milk production and the growth of their lambs, especially in the case of twins, were reduced when they received hay of poor quality.

The results concerning the estimated metabolisable energy balance, the mobilization of body reserves, the probable effects of the ambiant temperature and of the latness of the animals are discussed in relation to the litter size and the quality of the hay offered.

\section{RÉFÉRENCES BIBLIOGRAPHIQUES}

Bines J. A., I97I. Metabolic and physical control of food intake in ruminants. Symposium Proceedings, 30, II6-I 22.

Blaxter K. L., IVainman F. W., Wilson R. S., i961. The regulation of food intake by sheep. Anim. Prod, 3, 5 I-62.

Demarquilly C., Weiss P., i970. Tableaux de la valeur alimentaire des fourrages. Éd. S. E. I., I. N. R. A., 78000 Versailles.

Dole V. D., Meinertz H., i96o. Micro determination of long chain fatty acids in plasma and tissues. J. Biol. Chem., 235, 2595-2599.

Fenr P. M., Guessous, Tissier M., Sauvant 19., ig7r. La gestation de la Chèvre laitière : aspects alimentaires, p. $2 \mathrm{I} \mathrm{I}-222, I I^{\mathrm{e}}$ Conférence internationale de l'Elevage caprin. Tours (France). Ed. par I'Institut 'Technique de l'Élevage Ovin et Caprin. Paris.

lorbes J. M., I97o. The voluntary food intake of pregnant and lactating ruminants: a review. Br. Vet. J., 126, I-I I.

Foot-Janet Z., Greenhalgh J. F. 1)., r97o. A note on the relation between weights of alimentary tract contents, body fat and the uterus in Blackface ewes. Anim. Prod., 12, 669-67..

Foot-JANET Z., 1972. A note on the effect of body condition on the voluntary intake of dried grass wafers by Scottish Blackface ewes. Anim. Prod., 14, I3I-I34.

Hadjipieris G., Holmes W., I 966 . Studies on feed intake and feed utilization by sheep. I. The voluntary feed intake of dry, pregnant and lactating ewes. J. Agric. Sci. Camb., 66, $217-223$.

Johnson W. L., Trimberger G. W., Wright M. J., Van Vleeck L. 1)., Henderson C. R., 1966. Voluntary intake of forage by Holstein cows as influenced by lactation, gestation, body weight and frequency of feeding. J. Dairy Sci., $\mathbf{4 9}, 856$.

Journet M., Poutous M., Calomitr S., i 965 . Appétit de la Vache laitière. I. Variations individuelles de: quantités d'aliment ingérées. Ann. Zootech, , 14, 5-38.

Journet M., Jaririge R., I970. Facteurs physiologiques de ta quantité d'aliment ingérce par les Ruminants. $2 \mathrm{I}^{\mathrm{e}}$ réunion de la F. E. $Z$., I3udapest $24-28$ août 1970 .

MaKela A., I956. Studies on the guestion of bulk in nutrition of farm animals with special reference to cattle. Acta Agric. Genn, 85, I-I30.

McDonald M. A., Bell J. M., I958. Effect of low fluctuating temperatures on Farm animals, Part III. Can. J. Anim. Sci., 38, I48.

PEART J. N., r967. The effect of different levels of nutrition during late pregnancy on the subsequent milk production of Blackface ewes and on the growth of their lambs. J. A gric. Sci., 68, 365-37r.

PEART J. N., I97o. The influence of live weight and body condition on the subsequent milk production of Blackface ewes following a period of undernourishment in early lactation. J. agric. Sci., 75, 459-469.

Rattray P. V., (iarrett W. N., East N. E., Hinman N., I973. Net energy requirements of ewe lambs for maintenance, gain and pregnancy, and net energy values of feedstuffs for lambs. J.Anim. Sci., 37, 85.3-857. 
Rattray P. V., Garritt W. N., East N. E. linmann M., I974. Efficiency of utilization of metabolizable energy during pregnancy, and the energy requirements for pregnancy in sheep. J.Anim. Sci, 38, $383-393$.

Ricordeav G., Boccard R., Denamur R., ig60. Mesure de la production laitière des brebis pendant la période d'allaitement. Ann. Zootech., 9, 97-r 20.

Russel A. J. F., Donir J. M., ReID R. L., 1967. The use of biochemical parameters in controlling nutritional state in pregnant ewes, and the effect of under-nourishment during pregnancy on lamb birth weight. J. Agric. Sci., 68, 35 I-358.

Theriez M., Molenat G., I97o. Influence de lá taille du lot sur la quantité d'aliment ingérée par la Brebis en beryerie. Ann. Zootech., 19, 455-459.

Treacher T. T., r97o. Effects of nutrition in late pregnancy on subsequent milk production in ewes. Anim. Prod., 12, 23-36.

Treacher T. T., I97I. Effects of nutrition in pregnancy and in lactation on mills yield in ewes. Anim. Prod., 13, 493-501.

WALlace L. R., I948. The growth of lambs before and after birth in relation to level of nutrition. J. Agric. Sci., 38, 93 .

Winfield C. J., Brown W., Lucas I. A. M., I969. Sheltering behaviour at lambing by welsh Mountain ewes. Anim. Prod., 11, Ior-Iog.

Wodzicka-Tomaszewska M., i963. The effect of shearing of sheep. New Zealand J. Agric. Res., 6, 440-447. 